\title{
INTELLIGENTE LÖSUNGEN
}

Liebe Leserin, lieber Leser,

Nutzfahrzeuge erledigen rund drei Viertel der gesamten Güterverkehrsleistung in Europa - mit steigender Tendenz. Unser modernes Einkaufsverhalten wäre ohne die Lkw auf der Straße undenkbar. Hinzu kommt, dass alle anderen Verkehrsträger auf die logistische Unterstützung durch den Lkw angewiesen sind. Bevor also einfach eine Verlagerung von der Straße auf andere Verkehrsträger propagiert wird, sollte ein klares und zukunftsorientiertes Konzept zur sinnvollen Vernetzung der verschiedenen Verkehrsträger vorliegen.

Natürlich ist bei Nfz, ebenso wie bei Pkw, die Senkung von Verbrauch und Emissionen das Thema schlechthin. Und das aus zwei unterschiedlichen Perspektiven: Senkung der Betriebskosten für die Betreiber sowie Reduzierung der Emissionsbelastung für die Umwelt. Die Industrie entwickelt daher mit Hochdruck an intelligenten und zukunftsweisenden Lösungen. So werden auf der diesjährigen IAA neue, saubere Euro-VI-Aggregate ebenso zu sehen sein wie Innovationen zur Hybridisierung oder zur Wärmeenergierückgewinnung. Da dürfen natürlich auch Impulse zur Optimierung der Aerodynamik und zur Verringerung des Rollwiderstands nicht fehlen. Es zeigt sich wieder einmal, dass viele einzelne Maßnahmen zusammengenommen große Wirkung erzielen können. Getreu dem alten Motto der Lokomotivkonstrukteure: „Du kannst nirgendwo eine Tonne einsparen, aber an tausend Stellen ein Kilo."

Auch wenn gerade im Bereich Hybridisierung des Nutzfahrzeugs die Umsetzung in die Serie noch Zukunftsmusik ist, so ist doch gerade dieses Thema ein sehr vielversprechendes, da das Fahrprofil eines LKW erhebliches Potenzial zur Rekuperation und damit zum effizienten Einsatz der Hybridtechnologie in sich birgt. Knackpunkt wird hier vor allem die Wirtschaftlichkeit hybrider Systeme sein, denn für den Spediteur zählen heute Anschaffungspreis und Betriebskosten mehr denn je. Die Hersteller werden hier die richtigen Innovationen finden und auf die Straße bringen.

Die MTZ gibt in diesem Heft einen umfassenden Ausblick auf die aktuellen Innovationen. Ob Potenziale bei der Weiterentwicklung des Grundmotors, die neue Generation von Mercedes-BenzDieselmotoren für mittelschwere Nfz oder die Lösungen, die MAN Truck \& Bus im Köcher hat: Das Thema NutzfahrzeugEntwicklung ist spannender denn je.

Herzlichst, Ihr

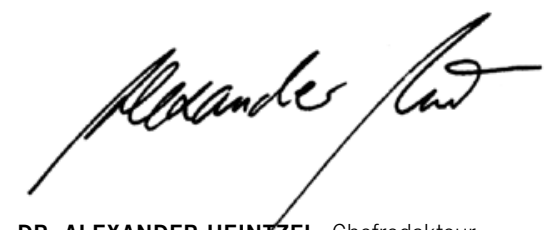

DR. ALEXANDER HEIN ZZEL, Chefredakteur Wiesbaden, 14. August 2012

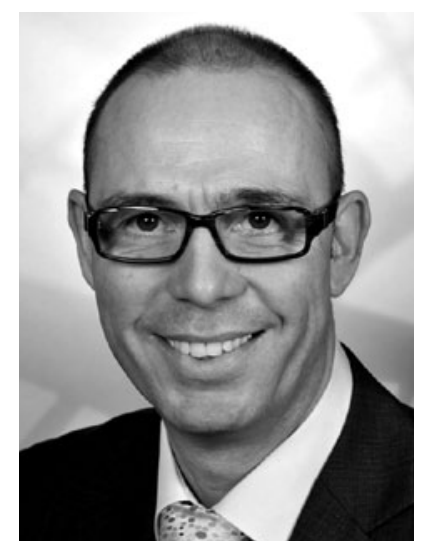

Mit

Simulation FreiZeit gewinnen

\section{Ihre Zeit-Vorteile}

- Umfangreiche Variantenvergleiche durchführen

- Schnell zu ersten Einschätzungen gelangen

Komplexe Fragestellungen transparent analysieren

\section{Für die Bereiche}

- Fahrleistung und Verbrauch

- Fahrerassistenzsysteme

- Fahrdynamik

- Motordynamik

Elektro- und Hybridfahrzeuge

\section{Mehr erfahren und am FreiZeit-Gewinnspiel teillnehmen}

www.tesis-dynaware.com/izb2012

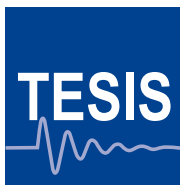

